\title{
Survivable Smart Grid Communication: Smart-Meters Meshes to the Rescue
}

\author{
Arjun P. Athreya and Patrick Tague \\ Wireless Network and System Security Group \\ Electrical and Computer Engineering \\ Carnegie Mellon University, USA \\ \{arjuna, tague\}@cmu.edu
}

\begin{abstract}
Smart grids are critical cyber-physical infrastructures in the world now. Since these infrastructures are prone to large scale outages due to disasters or faults, a resilient and survivable communication architecture is desired. In this work, we propose a resilient and survivable hierarchical communication architecture for the smart grid that mirrors the hierarchy of the existing power grid. Post-disaster resilience in grid communication is achieved through the grid flattening process. This process involves smart-meters and other disaster surviving elements of higher system levels of the grid forming a wireless mesh network. The flattened network of grid elements with one-hop communication links help in reliable and timely relaying of grid's health information to working regions of the grid. This allows for swift action by control engineers of the utility provider and emergency services with real-time data. We propose analytical models to study the performance of the flattened architecture as a function of outage area, smart-meter density and smart-meter's neighborhood size. The results from the analytical model will be compared with simulation results from OPNET.
\end{abstract}

\section{INTRODUCTION}

Integration of digital computing and communication technologies with the power-delivery infrastructure defines a smart grid, a critical cyber-physical infrastructure in today's modern world. The NIST conforming architecture [1] is designed to support control information sharing in the downstream links and measurement information from smart-meters in the upstream links. Recent research in smart grid communication and architecture have focused on evaluation of wireless technologies and use of cognitive radio and white space communication for the smart grids [2][3][4]. Focus has also been on control architecture for high assurance in smart grids [5] and RF mesh systems for smart metering and architectures have been studied for the smart grid [6]. However, the current smart grid architectures are not resilient to large scale blackouts and hence lose the ability to communicate [7].

In this paper, we propose a disaster resilient communication architecture using wireless mesh networks. On experiencing an outage due to a disaster, our proposed communication architecture reorganizes itself by the grid flattening process. Grid flattening process involves disaster surviving smartmeters and other working elements of the grid forming a wireless mesh network among themselves. Well established wireless mesh communication research [8] proves that efficient communication can be achieved even with battery operated transceivers.
We envision this communication architecture to mirror the existing power grid architecture and have multiple wireless mesh networks, each at a different system level. Bottommost layer of the architecture comprises of the customer locations equipped with smart-meters which communicate with a local distribution center. The local distribution centers then communicate with regional control centers which then communicate with Supervisory Control and Data Acquisition (SCADA) centers. At each of these system levels, wireless mesh networks are formed by grid elements communicating with their peers. When the system levels above the customer domain fail, smart-meters can form a wireless mesh network by communicating with their one-hop neighbors via the grid flattening process.

Advantages of this proposed architecture are, 1) Local and minor outage warning in a neighborhood can be quickly disseminated to other elements in the neighborhood through downstream communication in the hierarchy plus the neighboring areas through the wireless mesh, 2) Allows for control engineers to analyze data specific to areas seeing outages and could also collect health data shared with outage area's neighbors prior to the outage striking, 3) With intelligent advance warning systems in place, an area that could receive the spreading outage could be warned for evacuation or emergency services to be better prepared, or could also allow utilities providers for a graceful shutdown and 4) SCADA centers sharing data among themselves allows for health monitoring information to be shared across large areas. This allows for the grid to be gracefully shut down using the shared information.

Outage detection apart, the proposed architecture comes as a way to bootstrap a communication infrastructure that can help relay grid's critical health data. It is possible that grid's power line infrastructures are in place but with higher systems (local substations etc.) gone off-line. However, other externalities such as fire or flooding could make energy restoration hazardous even if higher system levels are functioning. With our proposed architecture, the data being sent across the network need not be only metering data, but could also be context specific data supplied by sensors on-board the smartmeter. These data could be local temperature, presence of flood water or even presence of toxic or flammable gases. All of these different kinds of data have an impact on the way the grid would function if there were automated self-healing 
procedures (if any in place) to restore energy in the grid. Safer and efficient decisions for acting on the grid could be made by control engineers or self-healing systems in remote locations with more features in grid's health data.

We present an analytical model to estimate the network performance of the flattened regions of the smart grid. We study the network performance as a function of smart-meter density, outage area size and smart-meter's neighborhood size. The results from the analytical model are compared with results from simulations for which OPNET [9] was used.

The remainder of this paper is organized as follows. Section II describes the architectural elements, the proposed architecture itself and interactions between the architectural elements. Grid flattening process is explained in Section III and Section IV illustrates performance analysis of the flattening process and the flattened network. We summarize our contribution in Section V.

\section{A COMMUNICATION ARCHITECTURE FRAMEWORK FOR THE SMART GRID}

With the motivation for a hierarchical communication architecture in Section I, we start this section by discussing the network assumptions. We then define the architectural elements and discuss the interactions between them.

\section{A. Network assumptions}

The assumptions made in this subsection facilitates the wireless communication abilities of smart-meters and other power grid's monitoring entities to form a wireless network.

1) The customer domain of the smart grid is divided in to logical neighborhoods with a local distribution center equipped with a transceiver. This transceiver has the capability to communicate wirelessly with individual smartmeters.

2) Smart-meters in radio proximity of each other have the ability to communicate with their peers via one-hop wireless links.

3) Smart-meters periodically update their peers in neighborhoods and the local distribution center.

4) Smart-meters are capable of sensing failure of elements in higher system levels of the power grid.

\section{B. Architecture elements and definitions}

We define the elements in our architecture that show the bottom-up approach we take to achieve survivability in the grid's communication. The architecture elements are shown in Figure 1.

- Micro-neighborhood: A micro-neighborhood $N_{i}$ is an area that consists of smart-meter equipped entities. The area of the micro-neighborhood depends on the density of homes or establishments in a geographical area equipped with smart-meters. The micro-neighborhood helps in defining the granularity level of the architecture to a specific smart-meter. This is the bottom-most layer in the proposed architecture. For ease in understanding and to avoid overlapping areas, each micro-neighborhood could

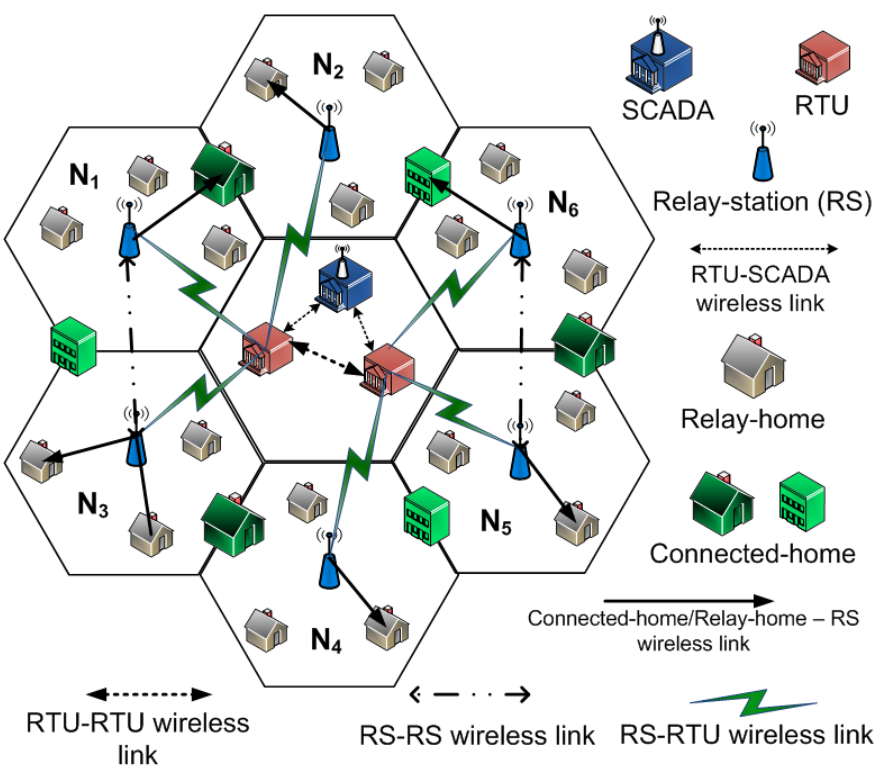

Fig. 1: This figure shows two macro-neighborhoods, each encompassing three micro-neighborhoods $\left(N_{1}, N_{2}, N_{3}\right)$ and $\left(N_{4}, N_{5}, N_{6}\right)$ respectively. The relay-stations form a wireless mesh network with their peers, and establish wireless links with RTU. RTUs form a wireless mesh network among their peers and establish wireless links with SCADA.

to be hexagonal in shape (closest approximation to a circle).

- Relay-home: A relay-home $R_{i}$ is a smart-meter equipped entity and belongs to a micro-neighborhood. The relayhome periodically reports its energy consumption to local distribution center(s) of the micro-neighborhood as programmed by the energy provider or the vendor of the smart-meter.

- Connected-home: A connected-home $C_{i}$ is a smartmeter equipped entity chosen in such a way that it is in radio proximity to at least two local distribution centers. For normal operations, a connected home is like any other relay-home reporting its energy consumption to the distribution center of the micro-neighborhood. However, a provision is made where a connected-home also reports to the distribution centers it can hear and establishes a communication link with the relay-homes in one-hop distance.

- Relay-station: A relay-station $R S_{i}$ is a transceiver that relays messages to and from all $R_{i} \mathrm{~s}$ and $C_{i} \mathrm{~s}$ of the micro-neighborhood to an aggregation entity such as the Remote Terminal Units (RTU). A relay-station can establish peer connections with relay-stations of adjacent micro-neighborhoods. Each micro-neighborhood has a local sub-station that distributes power to a neighborhood. Such a sub-station can be envisioned as a relay-station in our architecture.

- Macro-neighborhood: A macro-neighborhood $M_{i}$ is a collection of micro-neighborhoods. Each $M_{i}$ has an RTU 
that collects information and reports it to the SCADA for processing. Thus a collection of macro-neighborhoods can be a larger hexagonal area encompassing many $N_{i} \mathrm{~s}$, similar to the model used for cellular topology.

C. Hierarchical communication architecture for the smart grid with multiple overlaid wireless mesh networks

We envision this communication architecture to have multiple wireless mesh networks, and each at a different system level as shown in Figure 1. The bottom-most layer of grid is formed by the consumer domain consisting of $C_{i}$ s and $R_{i}$ s that communicate with a $R S_{i}$. The $R S_{i}$ s form the first and bottom most layer of the mesh network. The $R S_{i}$ s communicate with an RTU, and the RTU's form the second layer of meshnetwork in the architecture. Finally, RTU's communicate with SCADA and a collection of SCADA centers could form a wireless mesh spanning a larger area. This a very simplified model, there could be more division in system levels based on implementations. Each of these levels could follow the same communication hierarchy.

\section{Status codes}

We define status codes as numerical strings that convey to the elements the severity levels of the message. The status codes are listed in Table I in the increasing order of priority. Priority is given to the larger outage information to be propagated first and then smaller outages are acted upon. These numerical strings will help in aiding the process of grid flattening as we shall see in Section III-B.

TABLE I: Status code and their descriptions listed in increasing order of priority/severity level

\begin{tabular}{c|l}
\hline Status Code & Description \\
\hline 101 & ALL OK \\
\hline 191 & $\begin{array}{l}\text { LOCAL TROUBLE with ID of architecture entity facing } \\
\text { outage }\end{array}$ \\
\hline 201 & relay-home Failure \\
\hline 301 & connected-home Failure \\
\hline 401 & Micro-neighborhood failure \\
\hline 411 & Informing neighboring micro-neighborhood failure to RS \\
\hline 421 & Warning neighboring areas of micro-neighborhood failure \\
\hline 501 & Macro-neighborhood failure \\
\hline 511 & $\begin{array}{l}\text { Informing neighboring macro-neighborhood's failure to } \\
\text { RTU }\end{array}$ \\
\hline 521 & $\begin{array}{l}\text { Warning neighboring areas of macro-neighborhood fail- } \\
\text { ure }\end{array}$ \\
\hline 911 & Emergency, Immediate shut down and evacuation \\
\hline
\end{tabular}

We define a simple message format with key attributes that will not only convey status description, but also allow for actions with more detailed inputs. The notation $C \rightarrow R$ : $\left\langle M_{1}, M_{2}\right\rangle$ denotes an entity $C$ communicating with entity $R$ and conveying messages $M_{1}$ and $M_{2}$. An entity $\mathrm{C}$ broadcasting a message $\mathrm{M}$ is denoted by $C \rightarrow *:\langle M\rangle$. The identification tag linked to an entity such as connectedhome, relay-home etc is denoted as $I D_{\text {entity. }}$. Use of timestamp in messages will help control engineers understand and better study the behavior of certain entities if auditing is performed. The messages convey entities (ID affectedentity,
$\left.R S_{a f f e c t e d}\right)$ or neighborhoods ( $\left.M_{\text {affected }}, N_{\text {affected }}\right)$ facing minor outages or reporting minor anomalies. Fine grained location information is shared along with status codes which proves very effective for control and emergency operations. The format of the messages being shared can as follows, 〈Status Code, ID of neighborhood, ID of reporting entity, time-stamp, outage information $\rangle$.

\section{POST-DiSASTER SURVIVABLE COMMUNICATIONS VIA GRID FLATTENING}

Having defined the basic architecture and its elements, we now show how grid communication can survive an outage due to a disaster and thereby achieve resilience. We start this section by analyzing the after-effects of a series of failure in the power grid. We then discuss achieving resilience in communication after the disaster has struck.

Let us assume that a few $R S_{i}$ s see an outage due to some event such as fire or flooding, this means health monitoring information from smart-meters have no parent in the hierarchy to report the data. This sort of a failure leaves communication capable smart-meters in $R_{i}$ s and $C_{i}$ s to send out any critical measurement only via one-hop communication with their peers to an unaffected $R S_{i}$ which could then report to RTU and finally have emergency services to act swiftly. This clearly explains the need to have strategic placements of $C_{i} \mathrm{~s}$ that could help relay out critical data from affected regions of the grid to $R S_{i}$ of unaffected regions. This is possible because they are aware of one of the $R S_{i}$ failing and the other still normally operating, since a $C_{i}$ in the radio range to two $R S_{i} \mathrm{~s}$ can quickly inform its peers of a way to reach the unaffected region of the grid.

Relay-stations are not chosen as anchor nodes in establishing the communication links after a disaster for two reasons. First, if they are the local distribution substations they are more susceptible to go off-line first [7]. Second, they might not hear the neighboring relay-stations or RTUs if they also go off-line in large scale disasters. In this scenario, higher power and bandwidth are needed to support communications to reach the next available relay-station or RTU. However, with the use of connected-homes and relay-homes there could be delay in the control messages reaching the unaffected portion of the grid, but nonetheless it is more robust to link failures than depending on one centralized communication system.

\section{A. Grid flattening}

With $R S_{i}$ s failing first during an outage, it leaves the outage surviving smart-meters to communicate with their one-hop neighbors to relay out critical data to working regions of the grid by forming a wireless mesh network. This leads to a flat network formed by only the smart-meters. We call this process of establishing a survivable communication paradigm using only the metered entities as grid flattening as depicted in Figure 2. Even with the disaster spreading, this network formed by smart-meters can still survive and finally help data reach unaffected regions of the grid via multiple hops. 


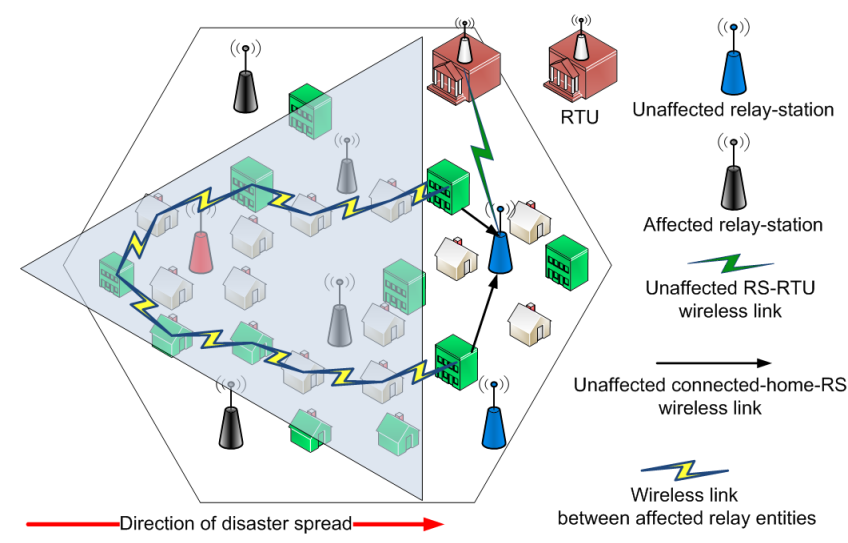

Fig. 2: This figure shows the outage facing area marked by the gray triangle of the smart grid is being flattened. The relayhomes and connected-homes form one-hop wireless links to create the flattened wireless mesh network.

\section{B. Communication among connected-homes and relay-homes}

When a connected-home senses the failure of the relaystation, it switches from the metering mode to the relay mode. The connected-home broadcasts the nature of the failure and a description with the status code 911 . When a relayhome or connected-home hears this message, it broadcasts this information further and warns its neighborhood of the imminent disaster as shown in Figure 3. This information propagates until it has seen a neighborhood that is not affected yet. Thus this model serves not only to propagate critical information but also warn their neighborhood of an impending disaster.

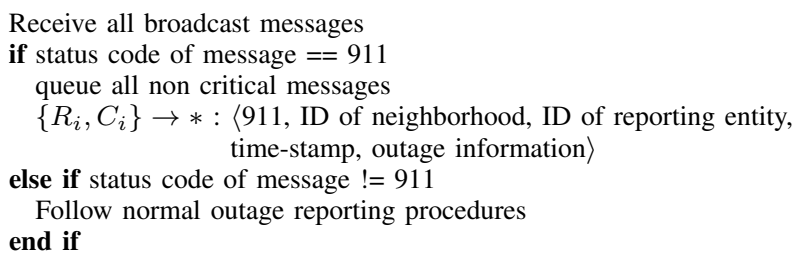

Fig. 3: Emergency status message processing at a relay-home or connected-home.

\section{PERFormance ANALYSis OF THE PROPOSED COMMUNICATION ARCHITECTURE FOR THE SMART GRID}

In this section, we provide a modeling framework for the communication architecture and compare the results from the modeling framework with the simulated results.

\section{A. Simulation setup}

Typically energy measurements are timed by the utility provider for usage data collection. Knowing the number of smart-meters to collect data from (a constant after an area is developed), a good collection schedule would suffice a graceful operation of the communication architecture even if a measurement is missed. Under normal operating conditions, the parent in each level of the hierarchy can function as the scheduler and decentralized scheduling can used for data collection among peers when the grid is flattened. For these discussed reasons, Time Division Multiple Access (TDMA) becomes the automatic choice for medium access eliminating the need for contention based medium access schemes.

We used OPNET's TDMA wireless modeler to simulate the proposed architecture. The simulations were carried out with different sizes of $N_{i}$ and with different transmission power of the smart-meters. Key parameters of the simulation setup are tabulated in Table II. The simulations were conducted over

TABLE II: Simulation fixed parameters

\begin{tabular}{c|c}
\hline Simulation Parameter & Value \\
\hline Base Frequency & $850 \mathrm{MHz}$ \\
\hline Channel Bandwidth & $10 \mathrm{MHz}$ \\
\hline TDMA Frame Length $f_{l}$ & $100 \mathrm{msec}$ \\
\hline TDMA Data slot length & $2 \mathrm{msec}$ \\
\hline TDMA SLots per Frame & 44 \\
\hline Maximum Data Payload per slot & 200 bytes \\
\hline Maximum data rate & $25 \mathrm{kbps}$ \\
\hline Packets per second & 50 \\
\hline Demand distribution & Uniform \\
\hline Simulation time & $0.1 \mathrm{hour}$ \\
\hline Radius of hexagonal area & $0.25 \mathrm{~km}$ \\
\hline Transmission range of smart-meters & $0.20 \mathrm{~km}$ \\
\hline
\end{tabular}

the following scenarios, 1) All neighborhoods are functioning correctly, 2) $N_{1}$ is flattened and $N_{2}$ and $N_{3}$ collect the critical data from $\left.N_{1}, 3\right) N_{1}$ and $N_{2}$ are flattened and $N_{4}$ and $N_{6}$ collect the critical data from $N_{1}$ and $N_{2}$ respectively and 4) One entire macro-neighborhood $\left(N_{1}, N_{2}, N_{3}\right)$ is flattened and $N_{4}$ and $N_{5}$ collect data from the affected macro-neighborhood.

\section{B. Modeling neighborhood density}

In this modeling framework we show the optimum number of smart-meters per neighborhood that suffices for the flattened network to gracefully survive the disaster and exhibit disaster resilience.

- Smart-meter density: The smart-meter density $\rho$ is the number of smart-meters per unit area. $\rho$ follows the distribution of a spatial Poisson process. This means that $\rho$ has a uniform density and the count of the smartmeters $N_{s m}$ has a Poisson distribution with mean $\rho A_{N_{i}}$. Since the current defined architecture has disjoint microneighborhoods, it follows that the count of smart-meters in micro-neighborhoods are also independent. Hence, this allows us to calculate the probability of $k$ smart-meters in a smaller disaster area $A_{\text {disaster }}$ of a micro-neighborhood with $n$ smart-meters, which follows a binomial distribution $\left(\begin{array}{l}n \\ k\end{array}\right) p^{k}(1-p)^{n-k}$ where $p$ is given by $\frac{A_{\text {disaster }}}{A_{N_{i}}}$.

- Smart-meter coverage area: Assuming an isotropic antenna on the smart-meter, the coverage area $A_{s m}$ is defined as the area spanned by the transmission signal of the smart-meter. 


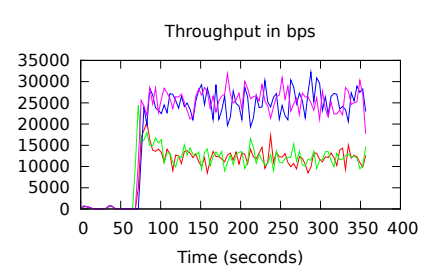

(a) $\rho=3$

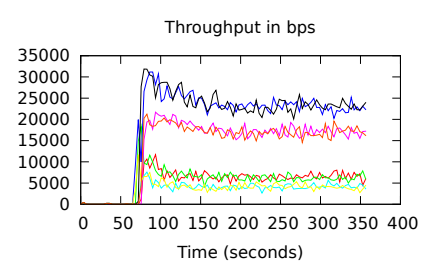

(e) $\rho=3$

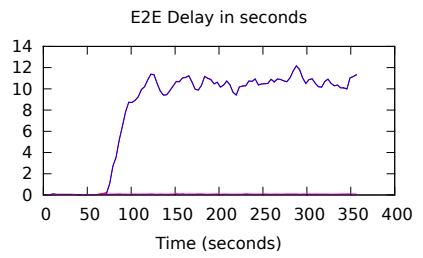

(b) $\rho=3$

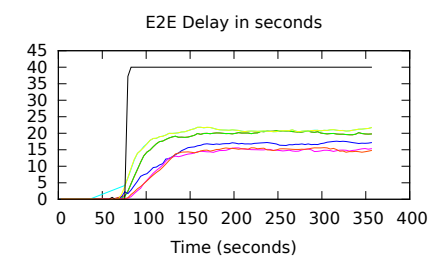

(f) $\rho=3$

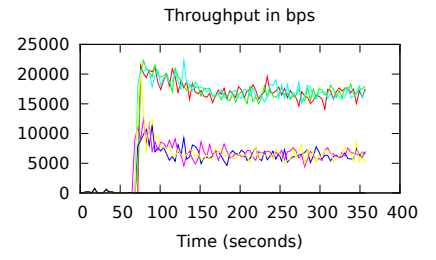

(c) $\rho=8$

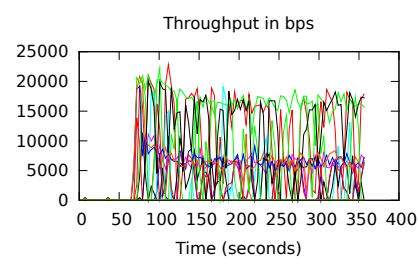

(g) $\rho=8$

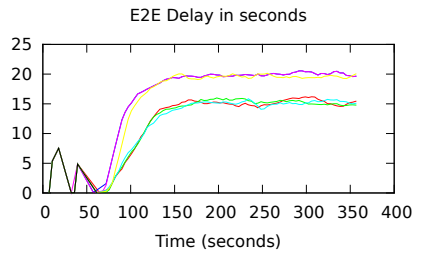

(d) $\rho=8$

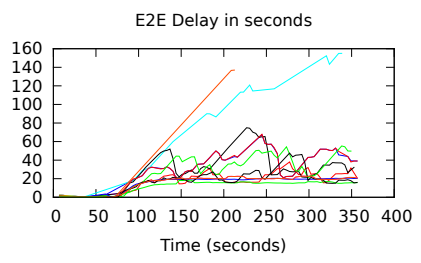

(h) $\rho=8$

Fig. 4: This figure shows throughput and delay performance when a macro-neighborhood is partially flattened with one microneighborhood's failure and completely flattened for $\rho=3$ in (a), (b), (e) and (f), and for $\rho=8$ in (c), (d), (g) and (h) respectively. Each line in these plots are measurements as seen in each of the smart-meter in the flattened micro-neighborhood(s).

- Effective smart-meter count: Given $\rho$ and $A_{s m}$, we define the effective smart-meter count $\eta_{\text {effective }}$ as $\rho A_{s m}$. This will indicate the number of smart-meters needed to cover the entire micro-neighborhood area. In reality, we would want this value to be greater than unity so that on an average, atleast 1 smart-meter is available to form a link to enable communication during the disaster.

Evaluation of smart-meter density: With simulation parameters as inputs, we calculate the minimum number of smartmeters needed to cover an entire micro-neighborhood. With the information from Table II, we see that $\eta_{\text {effective }}$ is 1.92 , as $A_{N_{i}}$ is $\pi(0.25)^{2}$ sq.km and $A_{s m}$ is $\pi(0.20)^{2}$ sq. km. We see that, on an average 2 smart-meters are heard for by every meter in the neighborhood leading to which 3 smart-meters would suffice to cover an entire micro-neighborhood.

$\eta_{\text {effective }}$ will be greater than 1 even for $\rho$ to be equal to 2. However, covering the same area with just 2 smart-meters means higher transmission power is needed. This shows that, atleast 3 smart-meters are required for the defined evaluation parameters. Hence we have arrived at an optimum number of smart-meters for $\pi(0.25)^{2}$ sq.km area needed to achieve network availability as far as the smart-meter as a device is concerned.

\section{Evaluation of throughput and delay as a function of smart- meter density}

In this subsection, we compare and analyze the performance of the flattened network in terms of throughput and delay with $\rho$ taking values 3 and 8 . Throughput in bps is defined as the amount of data that is successfully received at the network layer, and the end-to-end (E2E) delay is measured as time taken for demand to reach the destination from the source. From Figure 4, we clearly see a severe degradation in network performance when $\rho$ is equal to 8 . This shows that the throughput is not consistent and hence a particular node might not actually be available always for communication leading to the drastic increase in E2E delays. Analytical modeling explaining the drop in performance is discussed in Section IV-F.

The ideal behavior to characterize availability in this architecture is to have throughput degradation but with minimal variance around its mean. So, as long as there is consistent throughput in the flattened network, we are guaranteed of data being delivered at the cost of increased delay. This desired behavior is seen from the Figures $4 \mathrm{a}$ and $4 \mathrm{e}$ when $\rho$ is 3 .

\section{Scalability property}

We study scalability as a function of number of microneighborhoods failing. From Figure 5, we clearly see a graceful degradation in network performance, but not drastically dropping to an extremely low performance values that could render the flattened network unusable. Thus we show that even with an increase in micro-neighborhoods failing, we could still achieve a disaster survivable architecture. Figure 5 complements the results from Figures $4 \mathrm{a}$ and $4 \mathrm{e}$ which show network availability in both cases of one micro-neighborhood failing to all three micro-neighborhoods failing.

\section{E. Impact of TDMA neighborhood size on flattened network performance}

In the flattened network, smart-meters access the medium using a decentralized TDMA model. This means that for a given $\rho$ and flattened network area increasing, the number of TDMA neighbors sensed by each smart-meter is bound to increase. Impact of this increase is, lesser number of data slots that would be available for each contending smart-meter leading to fall in throughput at each of the smart-meters. 


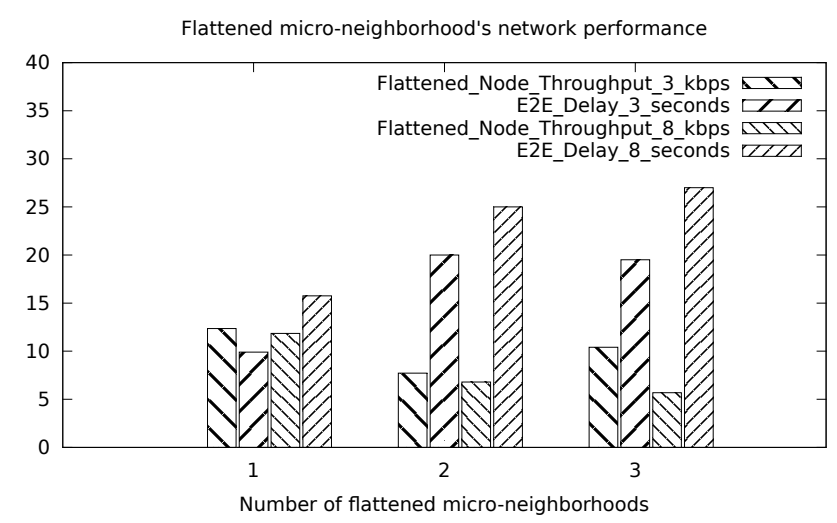

Fig. 5: This figure shows the network performance in terms of average throughput in kbps (Flattened_Node_Throughput_ $\left.\rho \_k b p s\right)$ and average end to end delay (E2E_Delay_ $\rho \_$seconds) in the flattened microneighborhoods and also for $\rho$ taking values 3 and 8. $N_{1}$ is flattened first, then $N_{1}$ and $N_{2}$ are flattened and finally $N_{1}$, $N_{2}$ and $N_{3}$ are flattened.

Network performance as a function of TDMA flattened neighborhood size from Table III is plotted in Figure 6. This figure clearly tells us that over provisioning the microneighborhood would lead to increasing in the number of TDMA neighbors seen by each smart-meter. This means that throughput will fall more rapidly and could bring down the network operation's efficiency when flattened. The throughput performance from Figure 6 also follow the scaling law as proposed in [10]. Hence, a definite trend in throughput performance degradation can be understood with increasing neighborhood size.

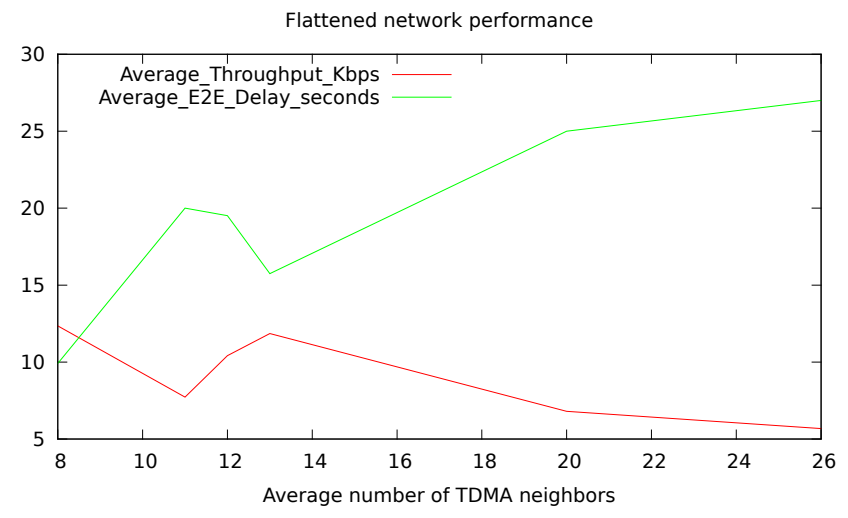

Fig. 6: This figure shows the network performance in terms of average throughput in kbps (Average_Throughput_kbps) and average end to end delay (Average_E2E_Delay_seconds) versus TDMA neighbor size
TABLE III: Evaluation of slot allocation expectation from the analytical model $\left(\mathrm{E}-n_{f}\right)$ to the results obtained from simulation $\left(\mathrm{R}-n_{f}\right)$

\begin{tabular}{c|c|c|c|c}
\hline Neighborhood(s) Flattened & $\rho$ & $N_{s m}$ & $\mathrm{E}-n_{f}$ & $\mathrm{R}-n_{f}$ \\
\hline$N_{1}$ & 3 & 8 & 5.5 & 6.3 \\
\hline$N_{1}, N_{2}$ & 3 & 11 & 4 & 4 \\
\hline$N_{1}$ & 8 & 12 & 3.67 & 4 \\
\hline$N_{1}, N_{2}, N_{3}$ & 3 & 13 & 3.38 & 3.25 \\
\hline$N_{1}, N_{2}$ & 8 & 20 & 2.20 & 1.68 \\
\hline$N_{1}, N_{2}, N_{3}$ & 8 & 26 & 1.69 & 1.58 \\
\hline
\end{tabular}

\section{F. Modeling TDMA slot allocation and impact on delay per- formance}

Given a TDMA frame, the data slots need to be shared by the contending smart-meters. In order to achieve reliability in the network performance, TDMA profile is designed to have 4 dedicated slots in each frame for each smart-meter. A probabilistic framework is developed in this subsection to estimate slots allocated to each smart-meter in each frame.

We start the problem formulation by defining some parameters that will help in arriving at the probabilistic framework. 1) $n_{f}$ : Data slots per frame, 2) i: A random variable that represents the number of slots a station gets in a frame and takes values $\left.\left[1: n_{f}\right], 3\right) N_{s m}$ : Number of smart-meters seen on average as neighbors by each of the smart-meter in the flattened grid and 4) $p$ : Probability of a smart-meter getting a slot in the frame. When the $N_{s m}$ smart-meters contend for a slot in the frame, the probability $p$ of a smart-meter getting that slot is $\frac{1}{N_{s m}}$. The contention for each subsequent slot is independent of the outcome of contending for a slot previously in the same frame. For a smart-meter to have gotten $\boldsymbol{i}$ slots in a frame, it has seen $\boldsymbol{i}$ successes and $n_{f}-\boldsymbol{i}$ failures.

Thus the probability of obtaining $\mathbf{i}$ slots in a frame is $p^{i} *(1-$ $p)^{\left(n_{f}-i\right)}$. Since the $\mathbf{i}$ slots can be in any order, the probability mass function of $\mathbf{i}$ is binomial. Hence the expectation of $\mathbf{i}$ becomes $n_{f} p$.

This analytical framework developed gives us the average number of slots a smart-meter gets per TDMA frame $n_{\text {slots }}$. The results from simulations and from the analytical model are tabulated in Table III, and we see about analytical model's results are $4 \%$ within simulated results.

Given that we have modeled the slot allocation mechanism, we now go a step further to model estimated value of delay. We now restructure the problem as, what is the expected number of frames that are needed to transmit a given quantity of data. This expectation now should also factor the loss seen in the physical layer that affects the throughput seen at the network layer. We assume that no delay to exist in data transfers between layers. Hence, the only delay seen is due to the transmission and data being queued for transmission denoted by $D_{q}$.

We start this exercise with the data rate of the sender D bps. We now model what is the expected number of frames required for $\mathrm{D}$ bits of data to reach the receiver. Let the data rate on an average be scaled by a factor of $\frac{1}{\sqrt{n_{s m} \log n_{s m}}}$, and 
lets call this scaled data rate as D' bps. Each smart-meter on an average gets $n_{f}$ slots per frame who's length is $100 \mathrm{msec}$. Thus in one second, a smart-meter gets $10 n_{f}$ slots on average. If D' bits are received in $10 n_{f}$ slots, the total number of slots needed to receive D bits is $\left\lceil\frac{10 n_{f} D}{D^{\prime}}\right\rceil$. Our problem statement now is to understand how many frames $k$ are needed to receive D bits factoring losses due to bit error in the channel given by $p_{e}$. Let the number of slots seen in $k$ frames be denoted by $N_{f}^{D}$.

$$
P\left[p_{e}\left(\sum_{j=1}^{k} n_{f}^{j}\right) \leq N_{f}^{D}\right] \leq \epsilon
$$

Using D as 25000 bps, $\epsilon$ as 0.005 and values of $n_{s m}$ from Table III, we tabulate $N_{f}^{D}, k$ and $D_{q}$ in Table IV. The probability of successful transmission $p_{e}$, we noticed that on an average $50 \%$ of the bits were in error as seen in simulation results and hence we use $p_{e}$ as 0.5 in estimating $k$. The average delay calculated as $k f_{l}+D_{q}$, is compared with the delay from the simulations and is plotted in Figure 7.

TABLE IV: Delay performance from TMDA's analytical model

\begin{tabular}{c|c|c|c|c}
\hline$n_{s m}$ & Slots/second & $N_{f}^{k}$ & $k$ & $D_{q}$ seconds \\
\hline 8 & 55 & 148 & 66 & 1.6 \\
\hline 11 & 40 & 136 & 84 & 2.38 \\
\hline 12 & 33.8 & 122 & 87 & 2.29 \\
\hline 13 & 36.7 & 140 & 106 & 2.8 \\
\hline 23 & 22 & 124 & 162 & 4.596 \\
\hline 26 & 16.9 & 102 & 155 & 5.06 \\
\hline
\end{tabular}

TDMA delay evaluation

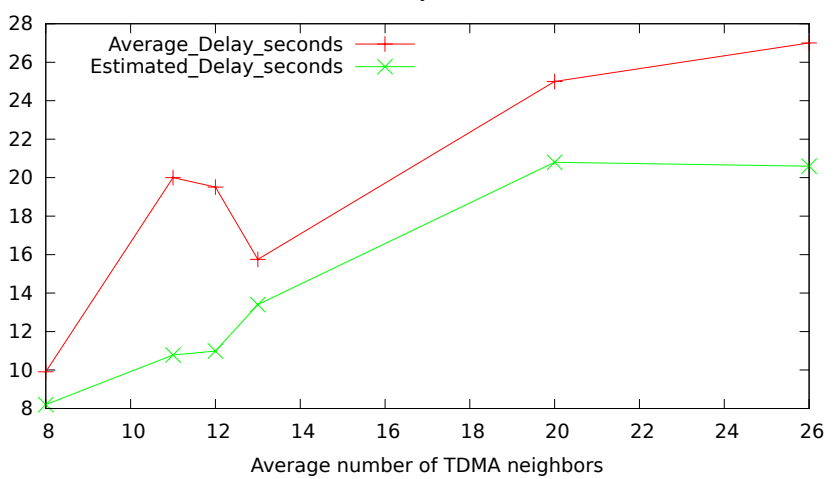

Fig. 7: This figure shows the comparison in delay as seen in the simulations with respect to the delay calculated from the TDMA's analytical model. The difference in analytical model's results to simulation results is due to the fact that we have not factored for all components that contribute to OPNET's modeling behavior.

We see that the analytical model performs equally well when compared to the simulated data. With this modeling framework, we have now provided a complete framework where a utility service provider can model the network performance and then accordingly plan the neighborhood and accordingly deploy the survivable communication architecture that supplements their current power grid.

\section{CONClusion}

We proposed a novel disaster survivable communication architecture for the smart grid was proposed. Our proposed architecture acts as an overlay on the existing power grid with post-disaster communication being facilitated by the grid flattening process. Timely and efficient dissemination of control data is possible through the hierarchical links and mesh networking at different system levels. We simulate this architecture using OPNET and use analytical models to study and compare the architecture's performance when the grid is flattened. Thus we have provided a complete framework to model a disaster survivable communication architecture for the smart grids. Simulated study results validate the results obtained from our analytical models. As future work, we will continue to develop the algorithms needed to flatten grid components using mesh networking fundamentals and make the architecture more scalable. We also intend to patch the difference in results from simulations and analytical models by further factoring in components that precisely model the OPNET modeler's behavior. As an interesting exercise, we also intend to setup a testbed of smart-meters that allow us to implement the grid flattening process we proposed.

\section{REFERENCES}

[1] "NIST Framework and Roadmap for Smart Grid Interoperability Standards, Release 1.0," Jan 2010, Office of the National Coordinator for Smart Grid Interoperability, http://www.nist.gov/public_affairs/releases/ upload/smartgrid_interoperabillity_final.pdf.

[2] A. Ghassemi, S. Bavarian, and L. Lampe, "Cognitive radio for smart grid communications," in Smart Grid Communications (SmartGridComm), 2010 First IEEE International Conference on, oct. 2010, pp. 297 -302.

[3] M. Souryal, C. Gentile, D. Griffith, D. Cypher, and N. Golmie, "A methodology to evaluate wireless technologies for the smart grid," in Smart Grid Communications (SmartGridComm), 2010 First IEEE International Conference on, oct. 2010, pp. $356-361$.

[4] R. C. Qiu, Z. Chen, N. Guo, Y. Song, P. Zhang, H. Li, and L. Lai, "Towards a real-time cognitive radio network testbed: Architecture, hardware platform, and application to smart grid," in Networking Technologies for Software Defined Radio (SDR) Networks, 2010 Fifth IEEE Workshop on, june 2010, pp. 1 -6.

[5] T. Overman and R. Sackman, "High assurance smart grid: Smart grid control systems communications architecture," in Smart Grid Communications (SmartGridComm), 2010 First IEEE International Conference on, oct. 2010, pp. $19-24$.

[6] B. Lichtensteiger, B. Bjelajac, C. Muller, and C. Wietfeld, "Rf mesh systems for smart metering: System architecture and performance," in Smart Grid Communications (SmartGridComm), 2010 First IEEE International Conference on, oct. 2010, pp. 379 -384.

[7] C. Hauser, D. Bakken, and A. Bose, "A failure to communicate: next generation communication requirements, technologies, and architecture for the electric power grid," Power and Energy Magazine, IEEE, vol. 3, no. 2, pp. 47 - 55, march-april 2005.

[8] I. Akyildiz and X. Wang, "A survey on wireless mesh networks," Communications Magazine, IEEE, vol. 43, no. 9, pp. S23 - S30, sept. 2005.

[9] “OPNET Modeler," http://www.opnet.com/solutions/network_rd/ modeler_wireless.html.

[10] P. Gupta and P. Kumar, "The capacity of wireless networks," Information Theory, IEEE Transactions on, vol. 46, no. 2, pp. 388 -404, mar 2000. 